\title{
IDENTIFICAÇÃO DE MODELOS VAR E CAUSALIDADE DE GRANGER: UMA NOTA DE ADVERTÊNCIA
}

\author{
Marco A. F. H. Cavalcanti *
}

\begin{abstract}
Resumo
O objetivo desta nota é alertar os leitores para um erro comum na literatura macroeconômica aplicada ao Brasil, associado à identificação de modelos VAR com base nos resultados de testes de causalidade de Granger.

Palavras-chave: Modelos autorregressivos vetoriais (VAR); Causalidade de Granger; Identificação
\end{abstract}

\begin{abstract}
In this note, we call attention to a popular mistake in the applied macroeconomics literature in Brazil - namely, the identification of VAR models based on the results of Granger causality tests.
\end{abstract}

Keywords: VAR models; Granger causality; Identification

JEL classification: $\mathrm{C} 32$

\section{Introdução}

A partir do artigo clássico de Sims (1980), o uso de modelos autorregressivos vetoriais (VAR) se difundiu rapidamente entre os economistas, e hoje esses modelos estão entre os instrumentos mais usados em investigações empíricas na área de macroeconomia. A popularidade dos modelos VAR deriva, em grande medida, da percepção de que tais modelos permitem analisar as interrelações entre múltiplas variáveis a partir de um conjunto mínimo de restrições de identificação - isto é, de restrições que permitam identificar o componente "exógeno" de cada variável, possibilitando a estimação do efeito de um "choque" nessa variável sobre as demais. Mesmo os adeptos dessa metodologia reconhecem, porém, que o problema de identificação está longe de ser resolvido - o que explica a coexistência de vários métodos alternativos de identificação na literatura. ${ }^{1}$

\footnotetext{
* Instituto de Pesquisa Econômica Aplicada - IPEA, Avenida Presidente Antônio Carlos 51/1407, Rio de Janeiro - RJ; e-mail: marco.cavalcanti@ipea.gov.br.

${ }^{1}$ Parte dos métodos baseia-se na imposição de restrições teóricas ou ad hoc sobre as relações contemporâneas entre as variáveis (Sims 1980, 1986, Bernanke 1986), sobre os efeitos de longo prazo de certos choques (Blanchard \& Fisher 1989) ou sobre os sinais dos efeitos dos choques (Uhlig 2005); outras abordagens buscam explorar propriedades dos dados, como as distribuições dos resíduos (Pesaran \& Shin 1998), quebras estruturais e heterocedasticidade (Lanne et al. 2009, Lanne \& Lutkepohl 2008) e correlações condicionais entre os resíduos do VAR (Swanson \& Granger 1997).
} 
Apesar de suas limitações, um dos métodos de identificação mais populares entre os macroeconomistas continua sendo o procedimento de ortogonalização dos resíduos do VAR com base na chamada "decomposição de Cholesky", que impõe uma estrutura recursiva à matriz de relações contemporâneas entre as variáveis do modelo - de modo que a primeira variável não seja afetada contemporaneamente por nenhuma das demais, a segunda seja afetada apenas pela primeira, a terceira seja afetada pelas primeiras duas, e assim por diante. Ao pesquisador cabe apenas selecionar a "ordenação causal" adequada das variáveis sob análise; feito isso, o modelo é exatamente identificado e é possível proceder à investigação das interrelações entre as variáveis por meio de funções de resposta a impulso e decomposição de variância dos erros de previsão do modelo.

Além da óbvia limitação de que a possibilidade de simultaneidade entre as variáveis é descartada a priori, uma das principais dificuldades desse método reside no fato de que todas as possíveis ordenações das variáveis do VAR são observacionalmente equivalentes, de modo que não é possível diferenciar entre elas com base em critérios estatísticos. Assim, a opção por determinada ordenação requer justificativas a partir de argumentos teóricos ou de conhecimento prévio acerca da natureza das relações contemporâneas entre as variáveis. Na maioria dos casos, porém, é difícil apresentar argumentos convincentes em favor de certa ordenação causal. Em análises de política monetária, por exemplo, é comum a hipótese de que o Banco Central não é capaz de observar a inflação e o nível de atividade correntes, de modo que tais variáveis não podem afetar contemporaneamente o instrumento de política monetária - devendo, assim, ser ordenadas após o instrumento de política monetária no VAR; essa é a abordagem de Leeper et al. (1996), dentre outros. Mas a hipótese alternativa, segundo a qual as variáveis de estado da economia podem afetar contemporaneamente o instrumento de política monetária, e não viceversa, também é utilizada em vários estudos; conforme assinalam Christiano et al. (1999), esta hipótese é pelo menos tão plausível quanto a anterior. Esse exemplo apenas ilustra a dificuldade geral do método, assim resumida por Demiralp \& Hoover (2003): "Only rarely does economic theory imply particular contemporaneous causal orderings. Generally, practitioners of SVAR methods appeal to plausible stories about which variables could or could not affect which other variables in the course of a month or quarter, depending on the periodicity of the data. The problem with this approach is that sometimes equally plausible stories can be told for competing causal orderings".

A insatisfação com certo grau inevitável de arbitrariedade na identificação de modelos VAR a partir de restrições teóricas tem levado alguns economistas a buscar métodos alternativos, nos quais a identificação esteja baseada pelo menos parcialmente em procedimentos estatísticos. Nesse contexto, vale destacar as contribuições de Pesaran \& Shin (1998), cujas funções de resposta a impulso "generalizadas" dependem da distribuição observada dos resíduos do VAR, de Swanson \& Granger (1997), que exploram as correlações condicionais dos resíduos do VAR com base na teoria dos grafos acíclicos direcionados de Spirtes et al. (2000), e de Lanne \& Lutkepohl (2008), que identificam o modelo a partir de mudanças na volatilidade dos choques ao longo da amostra. Tais métodos apresentam suas próprias dificuldades, ${ }^{2}$ mas, na pior das hipóteses,

\footnotetext{
${ }^{2}$ Para uma breve discussão de algumas das limitações desses métodos, ver Wallis (2004) acerca do problema de interpretabilidade de funções de resposta a impulso generalizadas, Ho-
} 
podem revelar-se complementos úteis aos métodos tradicionais.

Esse não é o caso, porém, de outro método de identificação que tem se revelado bastante popular no Brasil, baseado na realização de testes de causalidade de Granger (Granger 1969). De acordo com esse método, o VAR é identificado através de uma decomposição de Cholesky na qual a ordenação causal é definida em conformidade com os resultados de testes de causalidade de Granger, sob a hipótese implícita de que a ausência de causalidade (no sentido de Granger) de uma variável $X$ para outra variável $Z$ deve implicar a ausência de efeito contemporâneo de $X$ sobre $Z$. O objetivo aparente desse procedimento seria reduzir o grau de arbitrariedade na escolha da ordenação causal a ser adotada, pois esta estaria amparada em critérios estatísticos. Mas trata-se de um equívoco, pois o conceito de causalidade de Granger não guarda necessariamente nenhuma relação com a ocorrência (ou não) de efeitos contemporâneos de uma variável sobre outra.

O objetivo desta nota é alertar os leitores para esse erro comum. Inicialmente, mostra-se que o fato de certa variável $X$ não causar outra variável $Z$ no sentido de Granger não é condição necessária nem suficiente para a ausência de efeito contemporâneo de $X$ sobre $Z$. Essa conclusão depõe fortemente contra o método de identificação em questão, mas por si só não o invalida totalmente, pois a ausência de causalidade de Granger poderia estar positivamente correlacionada com a ausência de efeito contemporâneo de uma variável sobre outra - de modo que, na prática, o método poderia conduzir indiretamente a conclusões corretas acerca da ordenação adequada do VAR. Em uma segunda etapa, porém, a nota mostra que não há razão para crer que isso ocorra em macroeconomia, pois são prováveis situações em que uma variável $X$ tenha um efeito contemporâneo não nulo sobre $Z$, apesar de não causá-la no sentido de Granger. Tais resultados são apresentados no contexto de um VAR bivariado, mas são facilmente generalizáveis para modelos com três ou mais variáveis.

Cabe notar que os principais resultados apresentados nesta nota não são inéditos, podendo ser encontrados ou inferidos a partir de manuais de econometria de séries temporais ou artigos correlatos - ainda que não de forma sistematizada como se encontram aqui. Não obstante, parece importante esclarecer adequadamente a questão, pois o erro metodológico apontado acima tem se revelado muito comum na literatura macroeconômica aplicada ao Brasil: uma pesquisa não exaustiva da literatura recente detectou esse erro em 6 artigos publicados em importantes periódicos nacionais e em pelo menos outros 18 trabalhos - que incluem textos para discussão e textos apresentados em congressos, dissertações de mestrado e teses de doutorado. ${ }^{3}$ Vale ressaltar que muitos dos trabalhos citados justificam a adoção desse método equivocado de identificação com base em trabalhos anteriores que também o adotaram, o que indica elevado potencial de propagação do erro e reforça a relevância da publicação desta nota.

over (2001, cap. 7) e Céspedes et al. (2008) sobre as dificuldades de aplicação do método de Spirtes et al. (2000) à macroeconomia, e Lanne \& Lutkepohl (2008) a respeito das condições sob as quais o método por eles proposto seria válido.

${ }^{3}$ Ver, dentre outros: Albuquerque (2005), Bastos (2007), Belaisch (2003), Biage et al. (2003), Camuri (2005), Chinellato-Neto (2007), Costa (2006), Fonseca (2008), Klotzle (2008), Kobayashi (2008), Lamounier \& Nogueira (2007), Mendonça (2004, 2005), Neves et al. (2007), Oreiro et al. (2006), Resende (2006), Silva \& Resende (2008), Val \& Libâno (2009), Vartanian (2008) e Vieira \& Veríssimo (2005). 


\section{Identificação e causalidade em um VAR bivariado}

\subsection{VAR na forma reduzida e na forma estrutural}

Suponha, por simplicidade, um VAR(1) bivariado estacionário com forma estrutural dada por:

$$
\left[\begin{array}{cc}
1 & b_{12} \\
b_{21} & 1
\end{array}\right]\left[\begin{array}{l}
Z_{t} \\
X_{t}
\end{array}\right]=\left[\begin{array}{ll}
\gamma_{11} & \gamma_{12} \\
\gamma_{21} & \gamma_{22}
\end{array}\right]\left[\begin{array}{l}
Z_{t-1} \\
X_{t-1}
\end{array}\right]+\left[\begin{array}{l}
\varepsilon_{1, t} \\
\varepsilon_{2, t}
\end{array}\right],
$$

onde $\varepsilon_{1, t}$ e $\varepsilon_{2, t}$ são ruído branco. A correspondente forma reduzida é:

$$
\left[\begin{array}{l}
Z_{t} \\
X_{t}
\end{array}\right]=\left[\begin{array}{ll}
c_{11} & c_{12} \\
c_{21} & c_{22}
\end{array}\right]\left[\begin{array}{l}
Z_{t-1} \\
X_{t-1}
\end{array}\right]+\left[\begin{array}{l}
u_{1, t} \\
u_{2, t}
\end{array}\right],
$$

onde:

$$
\begin{array}{ll}
c_{11}=\frac{\gamma_{11}-b_{12} \gamma_{21}}{1-b_{12} b_{21}} ; & c_{12}=\frac{\gamma_{12}-b_{12} \gamma_{22}}{1-b_{12} b_{21}} ; \\
c_{21}=\frac{\gamma_{21}-b_{21} \gamma_{11}}{1-b_{12} b_{21}} ; & c_{22}=\frac{\gamma_{22}-b_{21} \gamma_{12}}{1-b_{12} b_{21}} ; \\
u_{1, t}=\frac{\varepsilon_{1, t}-b_{12} \varepsilon_{2, t}}{1-b_{12} b_{21}} ; & u_{2, t}=\frac{\varepsilon_{2, t}-b_{21} \varepsilon_{1, t}}{1-b_{12} b_{21}} .
\end{array}
$$

O VAR na forma reduzida resume as correlações observadas entre as variáveis de interesse e pode ser estimado sem problemas. Entretanto, na ausência de restrições adicionais, não é possível "recuperar" o VAR na forma estrutural a partir da forma reduzida. Este é o problema da identificação.

A identificação do VAR estrutural (1) pelo método da "decomposição de Cholesky" baseia-se na imposição de duas restrições à forma estrutural. Primeiro, supõe-se que os choques estruturais não sejam correlacionados contemporaneamente - ou seja, $E\left(\varepsilon_{1, t} \varepsilon_{2, t}\right)=0$. Segundo, supõe-se que um dos coeficientes $b_{12}$ ou $b_{21}$ seja zero, o que equivale a impor uma "ordenação causal contemporânea" entre $X$ e $Z$ : sob $b_{12}=0$, $Z$ não é afetada contemporaneamente por $X$, mas $X$ é afetada por $Z$; e sob $b_{21}=0$ ocorre o inverso. Com base nessas restrições, o modelo é exatamente identificado e é possível proceder à investigação das interrelações entre as variáveis por meio de funções de resposta a impulso e decomposição de variância dos erros de previsão do modelo.

\subsection{Causalidade de Granger}

Uma variável $X$ causa outra variável $Z$ no sentido de Granger se a observação de $X$ no presente ou no passado ajuda a prever os valores futuros de $Z$ para algum horizonte de tempo (Granger 1969).

No contexto do modelo VAR bivariado, o conceito de causalidade de Granger pode ser facilmente caracterizado em termos dos coeficientes da forma reduzida. Em particular, sob a hipótese de que os valores correntes e passados das variáveis incluídas no modelo contenham toda a informação relevante para a previsão de seus valores futuros, pode-se mostrar que uma variável $X$ causa outra variável $Z$ se, na equação de $Z$, algum dos coeficientes associados 
às defasagens de $X$ é diferente de zero; alternativamente, $X$ não causa $Z$ se todos esses coeficientes são nulos. ${ }^{4}$

No modelo acima, que inclui apenas uma defasagem das variáveis analisadas, a ocorrência de causalidade de Granger de $X$ para $Z$ pode ser facilmente testada a partir de um teste-t sobre $c_{12}$. A hipótese nula do teste é que $X$ não causa $Z$ :

$$
\mathrm{H}_{0}: c_{12}=0 .
$$

Analogamente, o teste da hipótese de não causalidade de $Z$ para $X$ baseia-se no teste-t para $c_{21}$.

\subsection{Identificação baseada em testes de causalidade de Granger}

Uma das principais dificuldades do método de identificação baseado na decomposição de Cholesky refere-se à escolha da "ordenação causal" das variáveis - ou, no contexto do modelo acima, à escolha do coeficiente a ser zerado na matriz de relações contemporâneas do VAR estrutural $\left(b_{12}\right.$ ou $\left.b_{21}\right)$.

O procedimento de identificação adotado em vários estudos recentes propõe escolher o coeficiente a ser zerado com base na realização de testes de causalidade de Granger entre $X$ e $Z$, sob a hipótese implícita de que a ausência de causalidade (no sentido de Granger) de uma variável para outra deve implicar também a ausência de efeito contemporâneo. Os testes de causalidade de Granger podem apontar quatro possibilidades: (i) causalidade unidirecional de $Z$ para $X$; (ii) causalidade unidirecional de $X$ para $Z$; (iii) causalidade bidirecional; (iv) ausência de causalidade em qualquer direção. De acordo com o método citado, o coeficiente a ser zerado seria $b_{12}$ sob (i) e $b_{21}$ sob (ii); nos casos (iii) e (iv) não fica claro o procedimento a ser adotado, mas, aparentemente, alguns autores determinam a "ordenação causal" com base nos p-valores dos testes de causalidade de Granger.

Esse método de identificação é, porém, claramente equivocado. Considere, por exemplo, o teste de causalidade de Granger de $X$ para $Z$. Note que a hipótese nula do teste pode ser definida em função dos parâmetros da forma estrutural:

$$
\mathrm{H}_{0}: c_{12}=\frac{\gamma_{12}-b_{12} \gamma_{22}}{1-b_{12} b_{21}}=0
$$

Evidentemente, o que se está testando é se $\gamma_{12}-b_{12} \gamma_{22}=0$, e não se $b_{12}=0$. A hipótese nula do teste pode ser rejeitada, ou não, independentemente de $b_{12}$ assumir um valor nulo ou diferente de zero. Logo, é fácil ver que a ausência de causalidade de Granger de $X$ para $Z$ não é condição necessária nem suficiente para a ausência de efeito contemporâneo de $X$ sobre $Z$ - e que, portanto, o método de identificação baseado em testes de causalidade de Granger está fundamentado em uma premissa falsa.

Essa conclusão depõe fortemente contra o método de identificação em questão, mas por si só não o invalida totalmente. De fato, note que a hipótese nula (3) seria verdadeira sob três situações básicas:

1. (a) $\gamma_{12}=0$ e $b_{12}=0$;

2. (b) $\gamma_{12}=0$ e $\gamma_{22}=0$;

\footnotetext{
${ }^{4}$ No contexto de um VAR com três ou mais variáveis a caracterização do conceito de causalidade é um pouco mais complicada, pois é possível que, mesmo que todos os coeficientes das defasagens de $X$ na equação de $Z$ sejam nulos, $X$ ajude a prever $Z$ (dois ou mais passos à frente) através de seu efeito sobre as demais variáveis do sistema. Ver Lutkepohl (2006).
} 
3. (c) $\gamma_{12}, b_{12}, \gamma_{22} \neq 0$, sendo $\gamma_{12}=b_{12} \gamma_{22}$.

Caso a não rejeição da hipótese nula estivesse associada, na maior parte dos casos, à ocorrência da situação (a) - ou seja, caso a ausência de causalidade de Granger estivesse positivamente correlacionada com a ausência de efeito contemporâneo de uma variável sobre outra -, então seria possível argumentar que, apesar de teoricamente inadequado, o método de identificação sob análise poderia conduzir indiretamente, na prática, a conclusões corretas acerca da ordenação adequada do VAR.

Entretanto, essa possível justificativa para o método apresenta pelo menos dois problemas. Primeiro, se, de fato, se verificassem as igualdades $\gamma_{12}=0$ e $b_{12}=0$, então $X$ não deveria ter nenhum efeito sobre $Z$, de modo que a resposta de $Z$ a um choque em $X$ deveria ser nula para qualquer horizonte de tempo; isso não é, porém, o que ocorre na maioria dos estudos que empregam esse método de identificação. ${ }^{5}$

Segundo, em macroeconomia são prováveis situações em que a condição (c) acima seja satisfeita, de modo que não parece haver razão para associar a ausência de causalidade de Granger predominantemente à condição (a) - e, portanto, à ausência de efeito contemporâneo de uma variável sobre a outra. De fato, conforme já advertido por Sims (1972) e repetido, dentre outros, por Hoover (2001, cap. 7), quando uma variável $X$ é manipulada de modo a controlar outra variável $Z$, é possível que $X$ afete $Z$ contemporaneamente, apesar de não causá-la no sentido de Granger.

Esse segundo problema pode ser ilustrado a partir de um exemplo simples, inspirado em um modelo macroeconômico bastante popular na literatura recente (Svensson 1997, Giordani 2004). Suponha que as relações entre a inflação $(\pi)$, o produto $(y)$ e a taxa de juros nominal $(i)$ sejam representadas pelas seguintes equações:

$$
\begin{gathered}
\pi_{t}=\alpha_{y} y_{t}+\pi_{t-1}+\varepsilon_{1, t} \\
y_{t}=\beta_{y} y_{t-1}-\beta_{r}\left(i_{t-1}-\pi_{t-1}\right)+\varepsilon_{2, t} \\
i_{t}=\phi_{y} y_{t}+\phi_{\pi} \pi_{t}
\end{gathered}
$$

onde (4) é uma "curva de Phillips", (5) é uma "curva IS" e (6) é a função de reação da Autoridade Monetária. Todos os parâmetros são supostos diferentes de zero.

Usando (6), pode-se eliminar $i$ de (5) e reescrever o sistema como um VAR bivariado, com forma estrutural dada por:

$$
\left[\begin{array}{cc}
1 & -\alpha_{y} \\
0 & 1
\end{array}\right]\left[\begin{array}{c}
\pi_{t} \\
y_{t}
\end{array}\right]=\left[\begin{array}{cc}
1 & 0 \\
\beta_{r}\left(1-\phi_{\pi}\right) & \beta_{y}-\beta_{r} \phi_{y}
\end{array}\right]\left[\begin{array}{c}
\pi_{t-1} \\
y_{t-1}
\end{array}\right]+\left[\begin{array}{c}
\varepsilon_{1, t} \\
\varepsilon_{2, t}
\end{array}\right]
$$

\footnotetext{
${ }^{5}$ Cabe ressaltar que, no contexto de um VAR com três ou mais variáveis, mesmo que todos os coeficientes de $X$ (contemporâneo e defasado) na equação de $Z$ sejam nulos, é possível que um choque em $X$ afete $Z$ através de seu efeito sobre as demais variáveis do sistema. Continua sendo verdade, porém, que se $X$ não causa $Z$ no sentido de Granger e não afeta nenhuma variável do sistema contemporaneamente (isto é, $X$ é a última variável na ordenação de Cholesky), então a resposta de $Z$ a um choque em $X$ deve ser nula para qualquer período de tempo.
} 
e forma reduzida dada por:

$$
\left[\begin{array}{c}
\pi_{t} \\
y_{t}
\end{array}\right]=\left[\begin{array}{cc}
1+\alpha_{y} \beta_{r}\left(1-\phi_{\pi}\right) & \alpha_{y}\left(\beta_{y}-\beta_{r} \phi_{y}\right) \\
\beta_{r}\left(1-\phi_{\pi}\right) & \beta_{y}-\beta_{r} \phi_{y}
\end{array}\right]\left[\begin{array}{c}
\pi_{t-1} \\
y_{t-1}
\end{array}\right]+\left[\begin{array}{c}
u_{1, t} \\
u_{2, t}
\end{array}\right]
$$

É fácil ver que, nesse sistema, $y$ tem um efeito contemporâneo não nulo sobre $\pi$, mas não vice-versa, de modo que um economista que desejasse estimar adequadamente as interrelações entre essas variáveis a partir de um VAR deveria usar a "ordenação causal contemporânea" $y-\pi$.

Mas suponha que o economista optasse por determinar a ordenação das variáveis a partir da realização de testes de causalidade de Granger, conforme proposto acima; a pergunta é: esse método poderia levar à ordenação correta das variáveis? Supondo que a taxa de juros seja administrada de modo a minimizar os desvios da inflação em torno de uma meta, a resposta é: não. De fato, seja a função objetivo da A.M. dada por:

$$
L=E_{t} \sum_{\tau=t}^{\infty} \delta^{\tau-t} \frac{\pi_{\tau}^{2}}{2} .
$$

onde $\delta$ é um fator de desconto intertemporal e supõe-se, por simplicidade, que a meta de inflação seja zero. Conforme mostrado por Svensson (1997) em contexto semelhante, a política monetária ótima requer que a taxa de juros em $t$ seja definida de modo a satisfazer a seguinte condição:

$$
E_{t} \pi_{t+1}=\bar{\pi}=0 .
$$

Usando (4) e (5), essa condição pode ser escrita como:

$$
\left(1+\alpha_{y} \beta_{r}\right) \pi_{t}+\alpha_{y} \beta_{y} y_{t}-\alpha_{y} \beta_{r} i_{t}=0
$$

Resolvendo para $i$, obtém-se a regra ótima de política monetária:

$$
i_{t}=\left(\frac{1+\alpha_{y} \beta_{r}}{\alpha_{y} \beta_{r}}\right) \pi_{t}+\left(\frac{\beta_{y}}{\beta_{r}}\right) y_{t}
$$

Logo, se a A.M. segue a regra ótima (12), os coeficientes $\phi_{y}$ e $\phi_{\pi}$ da equação (6) são dados por $\phi_{y}=\beta_{y} / \beta_{r}$ e $\phi_{\pi}=\left(1+\alpha_{y} \beta_{r}\right) /\left(\alpha_{y} \beta_{r}\right)$, e a forma reduzida do sistema, (8), pode ser reescrita como:

$$
\left[\begin{array}{l}
\pi_{t} \\
y_{t}
\end{array}\right]=\left[\begin{array}{cc}
0 & 0 \\
-1 / \alpha_{y} & 0
\end{array}\right]\left[\begin{array}{l}
\pi_{t-1} \\
y_{t-1}
\end{array}\right]+\left[\begin{array}{l}
u_{1, t} \\
u_{2, t}
\end{array}\right]
$$

Observa-se por (13) que $\pi$ causa $y$, mas $y$ não causa $\pi$ no sentido de Granger. Logo, se o economista determinasse a ordenação causal contemporânea das variáveis do VAR de acordo com os resultados dos testes de causalidade de Granger, ele optaria pela ordenação equivocada $\pi-y$.

\section{Conclusão}

Esta nota procurou alertar os leitores para um erro comum na literatura macroeconômica aplicada, associado à identificação de modelos VAR com base nos resultados de testes de causalidade de Granger. Espera-se que os esclarecimentos aqui apresentados evitem a ulterior propagação desse erro. 


\section{Referências Bibliográficas}

Albuquerque, C. R. (2005), Taxas de câmbio e inflação no Brasil: Um estudo econométrico, PhD thesis, UFRGS.

Bastos, F. A. S. (2007), Relações de causalidade entre retornos acionários, juros, atividade econômica e inflação: evidências para a américa latina, Master's thesis, IBMEC.

Belaisch, A. (2003), Exchange rate pass-through in brazil, Technical report, IMF.

Bernanke, B. S. (1986), Alternative explanations of the money-income correlation., Technical report, NBER.

Biage, M., Correa, V. P. \& Neder, H. D. (2003), 'Risco país, fluxos de capitais e determinação da taxa de juros no brasil: Uma análise de impactos por meio da metodologia vec', Revista Economia 9, 63-113.

Blanchard, O. J. \& Fisher, S. (1989), Lectures on Macroeconomics, Cambridge University Press.

Camuri, P. A. (2005), Dívida pública, política fiscal e restrição externa no brasil: 1994-2004, Master's thesis, CEDEPLAR/UFMG.

Chinellato-Neto, A. (2007), Relações entre crédito e crescimento econômico no Brasil 2000-2006, PhD thesis, Universidade Federal de Viçosa.

Christiano, L. J., Eichenbaum, M. \& Evans, C. (1999), Monetary policy shocks: what have we learned and to what end?, in 'Handbook of Macroeconomics', Elsevier Science/North Holland.

Costa, E. M. (2006), Estratégia comercial em um ambiente de oligopólio entre brasil e estados unidos: estudo do setor siderúrgico, Master's thesis, Universidade Federal da Paraíba.

Céspedes, B., Lima, E. \& Maka, A. (2008), 'Monetary policy, inflation and the level of economic activity in brazil after the real plan: stylized facts from svar models.', Revista Brasileira de Economia 62, 123-160.

Demiralp \& Hoover, K. (2003), Searching for the causal structure of a vector autoregression, Technical report, Oxford Bulletin of Economics and Statistics.

Fonseca, M. W. (2008), Mecanismos de transmissão da política monetária no Brasil: Uma análise pós-regime de metas de inflação, PhD thesis, UFPR.

Giordani, P. (2004), 'An alternative explanation of the price puzzle', Journal of Monetary Economics 51, 1271-1296.

Granger, C. (1969), 'Investigating causal relations by econometric models and cross-spectral methods', Econometrica 37, 424-438.

Hoover, K. (2001), Causality in macroeconomics, Technical report, Cambridge University Press. 
Klotzle, A. C. (2008), Os determinantes das taxas de juros brasileiras para títulos públicos pré-fixados de longo prazo, Master's thesis, PUC-Rio.

Kobayashi, T. C. (2008), Volatilidade da conta financeira do balanço de pagamentos: os casos do brasil, méxico, argentina e chile, Master's thesis, UFU.

Lamounier, W. M. \& Nogueira, E. M. (2007), 'Causalidade entre os retornos de mercados de capitais emergentes e desenvolvidos', Revista Contabilidade e Finanças 43, 34-48.

Lanne, M. \& Lutkepohl, H. (2008), 'Identifying monetary policy shocks via changes in volatility', Journal of Money, Credit and Banking 40, 1131-1149.

Lanne, M., Lutkepohl, H. \& Maciejowska, K. (2009), 'Structural vector autoregressions with markov switching', Journal of Economic Dynamics and Control.

Leeper, E., Sims, C. \& Zha, T. (1996), 'What does monetary policy do?', Brookings Papers on Economic Activity 2, 1-78.

Lutkepohl, H. (2006), New Introduction to Multiple Time Series Analysis, Springer.

Mendonça, H. F. (2004), 'Dívida pública e estabilidade de preços no período pós-real: Explorando relações empíricas', Estudos Econômicos 34, 345-368.

Mendonça, H. F. (2005), Metas para inflação e variáveis macroeconômicas: uma avaliação empírica, in 'Encontro Nacional da ANPEC'.

Neves, A. L., Paula, L. R. F., Silva, G. J. C., Oreiro, J. L. C. \& Amaral, R. Q. (2007), Por que o custo do capital no brasil é tão alto?, in 'Encontro Nacional da ANPEC'.

Oreiro, J. L., Paula, L. F., Ono, F. H. \& Silva, G. J. C. (2006), 'Determinantes macroeconômicos do spread bancário no brasil: teoria e evidência recente.', Economia Aplicada 10, 609-634.

Pesaran, H. \& Shin, Y. (1998), 'Generalized impulse response analysis in linear multivariate models', Economics Letters 58, 17-29.

Resende, J. L. (2006), Metas de inflação, câmbio flexível e autonomia de política monetária, Master's thesis, CEDEPLAR/UFMG.

Silva, G. J. C. \& Resende, M. F. C. (2008), Eficácia dos controles de capitais no brasil: abordagem teórica e empírica alternativa, in 'XIII Encontro Nacional de Economia Política'.

Sims, C. (1972), 'Money, income and causality', American Economic Review 62, 540-552.

Sims, C. (1980), 'Macroeconomics and reality', Econometrica 48, 1-48.

Sims, C. A. (1986), Are forecasting models usable for policy analysis?, Technical report, The Federal Reserve Bank of Minneapolis.

Spirtes, P., Clark, G. \& Richard, S. (2000), Causation, Prediction, and Search, MIT Press. 
Svensson, L. (1997), 'Inflation forecast targeting: implementing and monitoring inflation targets', European Economic Review 41, 1111-1146.

Swanson, N. R. \& Granger, C. (1997), 'Impulse response functions based on a causal approach to residual orthogonalization in vector autoregressions', Journal of the American Statistical Association 92, 357-367.

Uhlig (2005), 'What are the effects of monetary policy on output? results from an agnostic identification procedure', Journal of Monetary Economics 52, 381-419.

Val, V. C. \& Libâno, G. (2009), Liberalização versus vulnerabilidade: uma análise empírica da recente instabilidade financeira no brasil a partir do índice de fragilidade externa, in 'II Encontro Internacional da Associação Keynesiana Brasileira'.

Vartanian, P. (2008), Choques monetários e cambiais sob regimes de câmbio flutuante nos países membros do mercosul: há indícios de convergência macroeconômica?, in 'Encontro Nacional da ANPEC'.

Vieira, F. V. \& Veríssimo, M. P. (2005), Crescimento econômico de longo prazo na china: uma investigação econométrica, in 'Encontro Nacional da ANPEC'.

Wallis, K. (2004), 'Modeling regional interdependencies using a global errorcorrecting macroeconometric model', Journal of Business and Economic Statistics 22, 172-175. 